\title{
Increased Detection of Polymicrobial Infections by Sonication and Significance of Prosthesis Design and Fixation Technique in Cases of Low-Grade PJI, a Retrospective Study
}

\author{
Nils Haustedt ${ }^{*} \#$, Jorge Armando González Chapa ${ }^{2 *}$, Moritz Hentschke ${ }^{3}$, \\ Adrian Geovanni Rosas Taraco² \\ ${ }^{1}$ Clinic of Septic Bone and Soft-Tissue Surgery, Schön-Klinik Hamburg Eilbek, Hamburg, Germany \\ ${ }^{2}$ Departamento de Inmunologia, Facultad de Medicina, UANL, Monterrey, Mexico \\ ${ }^{3}$ Departement of Microbiology and Virology, Labor Fenner, Hamburg, Germany \\ Email: "nhaustedt@schoen-klinik.de, jorgechapag89@gmail.com, mhentschke@fennerlabor.de, adrian.rosastr@uanl.edu.mx
}

How to cite this paper: Haustedt, N., Chapa, J.A.G., Hentschke, M. and Taraco, A.G.R. (2020) Increased Detection of Polymicrobial Infections by Sonication and Significance of Prosthesis Design and Fixation Technique in Cases of Low-Grade PJI, a Retrospective Study. Open Journal of Orthopedics, 10, 269-288.

https://doi.org/10.4236/ojo.2020.1010028

Received: September 29, 2020

Accepted: October 19, 2020

Published: October 22, 2020

Copyright $\odot 2020$ by author(s) and Scientific Research Publishing Inc. This work is licensed under the Creative Commons Attribution International License (CC BY 4.0).

http://creativecommons.org/licenses/by/4.0/

\begin{abstract}
Introduction: Low-grade PJI remains a diagnostic dilemma in the medical community. It is mainly caused by low-virulent bacteria that cause chronic infection. This is mainly due to the formation of biofilms on the implant surface. This biofilm formation poses a diagnostic challenge that causes difficulties in the microbiological diagnosis of the infection by conventional culture methods. Sonication culture of implants has proven to be useful for biofilm forming bacteria. Therefore, we examined the evaluation of low-grade PJI using sonication culture methods and searched for influencing factors. The methods: Between October 2016 and November 2019 we retrospectively examined adult patients with suggestive clinical and laboratory findings of low-grade PJI. The patient's medical history, demographic information, prosthesis type, laboratory findings and length of hospital stay were collected. The results of the microbiological culture were divided into the results of the conventional preoperative and intraoperative culture method and the results of the sonication. In addition, laboratory chemical diagnostics of the joint punctures and CRP determination were performed. Additionally, the influence of prosthesis design and fixation technique, with and without cement, was investigated. The Results: Fifty-two patients with a mean age of 70.5 years were included in this study. The female population was more frequently affected. The most frequently affected joints were hip and knee. A higher percentage of pathogen detection was found at sonication with $60 \%$ of positive cultures, compared to $52 \%$ of intraoperative and $40 \%$ of preoperative
\end{abstract}

*These authors contributed equally: Nils Haustedt and Jorge Armando Gonzalez Chapa. 
conventional positive culture findings. The majority of patients were monomicrobial infections, with Staphylococcus epidermidis being the most frequently detected etiologic pathogen. The sonication culture was best able to detect a polymicrobial infection in $14 \%$ of cases compared to $6 \%$ of intraoperative and $4 \%$ of preoperative conventional cultures. Patients with a polymicrobial infection showed higher CRP values $(P=0.0172)$ and longer hospitalization $(P=0.0017)$ than monomicrobial infected patients. The results of the intraoperative conventional culture showed the highest congruence with the results of the sonication culture (concordance of 79\%) compared to the preoperative culture. An infectious histopathological classification confirmed the microbiological ultrasound findings in $69 \%$ of cases with an infectious category. Cemented fixation showed a trend towards less positive microbiological findings, and total hip arthroplasty showed the highest detection of bacteria by sonication culture compared to bicondylar surface knee replacement $(P=0.0072)$. Conclusion: The sonication culture proved to be an important microbiological diagnostic tool, especially for the detection of polymicrobial infections in cases of low-grade PJI. This method has an established place in the diagnosis of low-grade PJI. The prosthesis design and the cemented fixation technique seem to have an influence on the detection of bacteria.

\section{Keywords}

PJI, Low Grade Infections, Biofilm, Sonication

\section{Introduction}

Periprosthetic joint infection (PJI) is a serious and complex situation after arthroplasty. As stablished in a general consensus PJI is defined by presenting one of the following major criteria: two positive periprosthetic cultures with phenotypically identical organisms or a sinus tract communicating with the joint [1] [2]. $\mathrm{PJI}$ is also defined by just the presence 3 of the following minor criteria: elevated serum CRP, ESR, synovial fluid WBC, ++ change on leukocyte esterase strip test, elevated synovial fluid PMN\%, positive histological analysis and a single positive culture [1] [2]. PJI might be usually classified depending on the time of onset as acute (high grade) or chronic (low grade) infections [3]. High grade infections usually display classical sings of an acute establishment with no further diagnostic difficulties, on other hand, low grade infections represent a real medical diagnostic challenge due to the presence of ambiguous clinical and laboratory findings [4]. Low grade infections usually are produced by low-virulent bacteria which cause a delayed postoperative onset [3] [5]. However, the persistence of the infection and the inflammatory process might be also related to special bacteria properties which make them to adhere and assemble in an enclosed extracellular matrix on implanted material, a biofilm [6]. This might cause resistance not only to host's endogenous immune response but also to drugs treatment in 
multiple ways which have been reviewed before [7] [8]. An unclear diagnosis is also often displayed due to negative microbiological cultures, in which prevalence has been reviewed to be between $5 \%$ and $42 \%$ [9]. The absence of microorganisms identification might be also attributable to biofilms, situation in which colonies are not produced from the samples taken and cultured into conventional growth methods [10]. Due to this fact, relatively new culturing methods have been tried to be able to increase the diagnostic reliability of infections, among them, the sonication culture through sound waves in the ultrasound spectrum mechanically removes adhered bacteria in the biofilms, on the implanted prosthetics. This technique has been represented as a simple and sensible method to improve the microbiologic diagnosis of PJI [11] [12]. A consistent and uniform definition for low grade PJI condition is still missing nowadays, which hinders a specific diagnostic protocol to follow [13]. In consequence, an attempt is to improve the diagnostic reliability multiple tests which have been taken as nonspecific parameters. However, microbiological findings are still considered as one of the most important for an accurate diagnosis. Due to these, we retrospectively analyzed the benefits of sonication culture in low grade PJI patients.

\section{Methods}

\subsection{Source Population}

We retrospectively reviewed the electronic health records of all diagnosed patients with low grade PJI at our institution (Schön Klinik Eilbek, Hamburg, Germany) from October 4, 2016 to November 1, 2019. Patients with a delayed or chronic postoperative onset, $\geq 4$ weeks after surgery, with $\geq 3$ weeks of symptoms and clinical-laboratory findings suggesting infection were included. All patients were scheduled for prostheses treatment removal in our institution. Exclusion criteria consisted in patients with an acute onset of the infection or an incomplete medical record. Demographic characteristics of the patients such as, age and gender were included. Information about prostheses type, affected anatomic region and hospitalization time were also collected. Laboratory results as blood $\mathrm{C}$-reactive protein (CRP) and leukocytes levels were reviewed. Pre-operation, intra-operation and sonication samples culture results were also recorded. Krenn classification histopathological analysis [14] of intraoperation samples was collected for the data base when available. The evaluated data of our study was anonymized, and the information was only accessible to the treatment and research team.

\subsection{Culturing Techniques}

\subsubsection{Sonication}

Explanted prostheses were transported in sterile boxes into the laboratory, where they were overlaid with phosphate buffered saline (PBS) and then shaken for 30 seconds. Afterwards they were sonicated for 3 minutes at $100 \%$ power in an ul- 
trasound bath (Bactosonic). Immediately after sonication the box was again vigorously shaken for 30 seconds, before removing $50 \mathrm{ml}$ of the sonication fluid into a $50 \mathrm{ml}$ tube. This tube was the centrifuged for 5 minutes at $5000 \mathrm{rpm}$. After centrifugation the supernatant was discarded, except $0.5 \mathrm{ml}$. The pellet was resuspended and then $100 \mu \mathrm{l}$ were plated each on a Columbia blood agar, a chocolate agar and a Schaedler agar (all from Becton Dickinson). The former two were incubated at $37^{\circ} \mathrm{C}$ and $5 \% \mathrm{CO}_{2}$ for 14 days, while the latter one was incubated anaerobically for 14 days. Finally, $100 \mu \mathrm{l}$ of the sonication fluid was transferred to a semifluid Leber agar (Sifin) enrichment medium, which was incubated at $37^{\circ} \mathrm{C}$ for 14 days as well. Plates and Leber-agar were inspected for growth on day 1 and 2 and then every three days. Bacteria were identified using a Maldi-Tof instrument (Bruker biotyper) and susceptibility tests were carried out using a Vitek 2 automation (Biomerieux).

\subsubsection{Standard Culture Methods}

Swabs were put in $2 \mathrm{ml}$ of physiological $\mathrm{NaCl}$ solution for up to 30 minutes, which was then streaked out on a blood agar plate (BD). Additionally, $400 \mu \mathrm{l}$ each were then transferred to a Brain-Heart-Infusion liquid medium (BHI, Becton Dickonson) and a Leber/Thioglycolat semifluid medium (Sifin). Excised tissue samples were homogenized and then transferred into BHI, Leber agar and Leber/Thioglycolat semifluid medium. Joint aspirates were centrifuged when appropriate (more than $3 \mathrm{ml}$ ) with removal of supernatant more than $3 \mathrm{ml}$ or used. The synovial fluid was streaked out on Columbia blood agar, transferred into BHI, Leber agar and Leber/Thioglycolat semifluid medium.

All materials were incubated at $37^{\circ} \mathrm{C}$ for 14 days. Plates and fluid media were inspected for growth on day 1 and 2 and then every three days. Bacteria were identified using a Maldi-Tof instrument (Bruker biotyper) and susceptibility tests were carried out using a Vitek 2 automation (Biomerieux).

\section{Analysis}

Medical records information was converted into structured data. Based on the time and methods in which the microbiological analysis was made results were divided into 3 groups: 1) pre-operative, 2) intra-operative and 3) sonication group. In group 1 and 2 standard culture results were examined. These results were compared with the sonication culture information (group 3) in terms of positive or negative findings. The specific microorganism was identified, and the number of microorganisms were found and also described. Descriptive statistics was used for determining the pathogen detection percentages and concordance of the different cultures results. Furthermore, we compared the different pathogen detection rates between the groups using Fisher exact test and Mann Whitney non-parametric Gaussian distribution test was used between 2 groups of data when possible. A $P$ value of $<0.05$ was considered as statically significant using Prism GraphPad 8 (San Diego, California). 


\section{Results}

\subsection{Demographic and Clinical Characteristics of the Patients}

As shown in Table 1 a total of 52 patients were enrolled in this study. Female patients had almost twice prevalence than male. The mean age of the patients was of 70.5 years old, both men and women patients presented no age differences with almost the same mean age, 71.2 and 70 years old respectively. The most common affected joints were the hip and the knee with the same number of cases, $44.23 \%$, followed by the shoulder with $11.53 \%$. Male patients most common affected joint were the hip with 55\% of the cases followed by the knee with $30 \%$ and shoulder in $15 \%$. On the other hand, females were more commonly affected in the knee with $53.12 \%$, followed by hip with $37.50 \%$ and shoulder $9.38 \%$. The inpatient length stay presented no differences in both groups of patients with almost a mean of 20 days. However, male patients showed a wider range of hospitalization days compared to women, 10 - 60 and 11 - 28 days respectively. Cemented type fixations were the most commonly used, this with a $63.46 \%$ of the cases compared to $34.61 \%$ of fixation without cement. Table 2 summarizes the prosthetics types used in the patients.

\subsection{Sonication Culture showed a Higher Percentage of Pathogen Detection}

Our results showed that the highest percentage of pathogen detection was found

Table 1. Patients characteristics.

\begin{tabular}{ll}
\hline Patients population data & \\
\hline $\mathrm{n}$ & 52 \\
Male & $20(38.5 \%)$ \\
Female & $32(61.5 \%)$ \\
Mean age in years (SD) [range] & $70.5(12)[37-89]$ \\
Male mean age (SD) [range] & $71.2(11)[43-85]$ \\
Female mean age (SD) [range] & $70(12)[37-89]$ \\
Inpatient length mean in days (SD) [range] & $19(7)[10-60]$ \\
Male mean in days (SD) [range] & $20(10)[10-60]$ \\
Female mean in days (SD) [range] & $19(5)[11-28]$ \\
Affected joints & Hip - 2 Knee - $23(44.23 \%)$ \\
& $3(44.23 \%)$ \\
& Shoulder- $6(11.53 \%)$ \\
Male & Knee -6 (30\%) \\
& Hip - 11 (55\%) \\
Female & Shoulder- 3 (15\%) \\
& Knee - $17(53.12 \%)$ \\
& Hip - $12(37.50 \%)$ \\
& Shoulder- $3(9.38 \%)$ \\
\hline
\end{tabular}


in the sonication culture with $60 \%$ of positive and $40 \%$ of negative cultures. On the other hand, the traditional cultures made with the intra-operative sampling showed a $52 \%$ of positive results while a $48 \%$ remained negative for microbiological analysis. The lowest percentage of bacterial detection was found in the pre-operative culture results with just a $40 \%$ of positive cultures. Table 3 resumes the culture percentages results of the 3 different groups.

\subsection{Staphylococcus Epidermidis Was the Most Common Pathogen Detected}

For most of the patients in the three groups just one etiological agent was identified as shown in Table 4. The most common isolated pathogen was Staphylococcus epidermidis with a $13 \%$ pre-operatively, $23 \%$ intra-operatively and $19 \%$ in sonication cultures. Table 5 shows that most of the cases detected with this etiological agent where during the intra-operative sampling. This, in contrast

Table 2. Prosthetics types in selected patients.

\begin{tabular}{|c|c|c|c|}
\hline \multicolumn{4}{|c|}{ Prosthetics type } \\
\hline Prosthetics type & All patients & Male patients & Female patients \\
\hline Bicondylar surface knee arthroplasty & $16(30.76 \%)$ & $5(25 \%)$ & $11(34.37 \%)$ \\
\hline Totalarthroplasties & $35(67.30 \%)$ & $14(70 \%)$ & $21(65.62 \%)$ \\
\hline Cement & $33(63.46 \%)$ & $11(55 \%)$ & $22(68.75 \%)$ \\
\hline Cementless & $18(34.61 \%)$ & $8(40 \%)$ & $10(31.25 \%)$ \\
\hline Bicondylar surface knee arthroplasty/ with cement & $13(25 \%)$ & $3(15 \%)$ & $10(31.25 \%)$ \\
\hline Bicondylar surface knee arthroplasty/ without cement & $2(3.84 \%)$ & $1(5 \%)$ & $1(3.12 \%)$ \\
\hline Total arthroplasties/ with cement & $19(36.53 \%)$ & $7(35 \%)$ & $12(37.50 \%)$ \\
\hline Total arthroplasties/ without cement & $16(30.76 \%)$ & $7(35 \%)$ & $9(28.12 \%)$ \\
\hline
\end{tabular}

${ }^{*} 1$ patient without information.

Table 3. Positive and negative microbiological cultures.

\begin{tabular}{ccc}
\hline & Patients cultures results & \\
\hline Pre-Op & Negative & Positive \\
Intra-Op & $31(60 \%)$ & $21(40 \%)$ \\
Sonication & $25(48 \%)$ & $27(52 \%)$ \\
\end{tabular}

Table 4. Monomicrobial and polymicrobial culture results.

\begin{tabular}{cccc}
\hline \multicolumn{3}{c}{ Patients cultures results } \\
\hline Monoinfection & Coinfection & Total positive cultures \\
\hline Pre-Op & $19(36 \%)$ & $2(4 \%)$ & $21(40 \%)$ \\
Intra-Op & $24(46 \%)$ & $3(6 \%)$ & $27(52 \%)$ \\
Sonication & $24(46 \%)$ & $7(14 \%)$ & $31(60 \%)$ \\
\hline
\end{tabular}


with Propionibacterium acnes which besides the fact that was the second most common detected pathogen in all groups it represented the most common agent detected in the sonication culture with a $17 \%$ of the cases. Staphylococcus aureus was found in $4 \%$ pre-operatively and in $6 \%$ intra-operatively. However, it was no detected in the sonication culture. Table 5 resumes the monomicrobial infective agents found in the three different studied groups. A positive culture result was only found in 5 cases (9.6\%) in sonication, while the other two groups remained with a negative culture result, while in the case of intraoperative samples only 1 patient (2\%) was positive and pre-operatively in 3 cases (5.7\%) as shown in $\mathrm{Ta}$ ble 6. Supplementary Table S1 shows all the microbiological findings and antibiogram in the three different groups.

\subsection{Sonication Culture Presented the Highest Coinfection Percentage Detection}

Multiple etiological agents were more commonly found in the sonication culture with a $14 \%$ of the cases compared to $4 \%$ pre-operatively and $6 \%$ intra-operatively, Table 4. Staphylococcus epidermidis was found as a co-infective agent in the three different groups while Staphylococcus aureus was just detected in the sonication group as shown in Table 7.

Table 5. Monomicrobial microorganisms found in cultures.

\begin{tabular}{cccc}
\hline & Pre-Op & Intra-Op & Sonication \\
\hline Staphylococcus epidermidis & $7(13 \%)$ & $12(23 \%)$ & $10(19 \%)$ \\
Propionibacterium acnes & $5(9 \%)$ & $4(7 \%)$ & $9(17 \%)$ \\
Staphylococcus aureus & $2(4 \%)$ & $3(6 \%)$ & - \\
Streptococcus faecalis & $2(4 \%)$ & - & $1(2 \%)$ \\
Staphylococcus capitis & $1(2 \%)$ & - & $1(2 \%)$ \\
Staphylococcus haemolyticus & - & - & $1(2 \%)$ \\
Streptococcus sanguinis & - & - & - \\
Pseudomona aureginosa & $1(2 \%)$ & $2(4 \%)$ & - \\
Streptococcus mitis & $1(2 \%)$ & $1(2 \%)$ & - \\
Actiomyces israelii & - & $1(2 \%)$ & $1(2 \%)$ \\
Staphylococcus lugdunensis & - & $3(6 \%)$ & $7(14 \%)$ \\
Co-infection & $2(4 \%)$ & $1(2 \%)$ & -
\end{tabular}

Table 6. Positive cultures when other groups remained with a negative culture.

\begin{tabular}{ccc}
\hline Pre-Op (5.7\%) & Intra-Op (2\%) & Sonication (9.6\%) \\
\hline Propionibacterium acnes & Staphylococcus epidermidis & Propionibacterium acnes \\
Staphylococcus epidermidis & Staphylococcus haemolyticus \\
Enterococcus faecalis & Propionibacterium acnes \\
& Propionibacterium acnes \\
& Propionibacterium acnes
\end{tabular}




\subsection{Multiple Infective Agents Associated with Higher CRP Levels and Longer Hospital Stay}

As shown in Figure 1(a), CRP serum levels of patients with multiple identified etiological agents were almost 4 times higher $(P=0.0172)$ with a mean of 79.99 $\mathrm{mg} / \mathrm{L}$, when compared to patients with just one infective agent who presented a mean of $20.50 \mathrm{mg} / \mathrm{L}$, this on sonication culture. Additionally, Figure 1(b) demonstrates a longer inpatient time care of almost 5 more hospitalization days in those patients with multiple pathogen infection detected with the same type of

Table 7. Polymicrobial microorganisms found in cultures.

\begin{tabular}{|c|c|c|}
\hline Pre-OP & Intra-OP & Sonication \\
\hline Staphylococcus epidermidis & Enterococcus faecalis & Staphylococcus aureus \\
\hline Staphylococcus caprae & Staphylococcus capitis & Staphylococcus caprae \\
\hline- & Staphylococcus warneri & - \\
\hline Pseudomonas aeruginosa & Staphylococcus epidermidis & Staphylococcus epidermidis \\
\hline Klebsiella aerogenes & Streptococcus sanguinis & Staphylococcus haemolyticus \\
\hline- & $\begin{array}{c}\text { Staphylococcus capitis } \\
\text { Propionibacterium acnes }\end{array}$ & $\begin{array}{l}\text { Staphylococcus epidermidis } \\
\text { Propionibacterium avidum }\end{array}$ \\
\hline- & - & $\begin{array}{c}\text { Staphylococcus aureus } \\
\text { Bacillus cereus }\end{array}$ \\
\hline- & - & $\begin{array}{c}\text { Pseudomonas aeruginosa } \\
\text { Staphylococcus haemolyticus }\end{array}$ \\
\hline- & - & $\begin{array}{c}\text { Staphylococcus aureus } \\
\text { Streptococcus oralis }\end{array}$ \\
\hline- & - & $\begin{array}{c}\text { Propionibacterium acnes } \\
\text { Actinomyces israelii }\end{array}$ \\
\hline
\end{tabular}
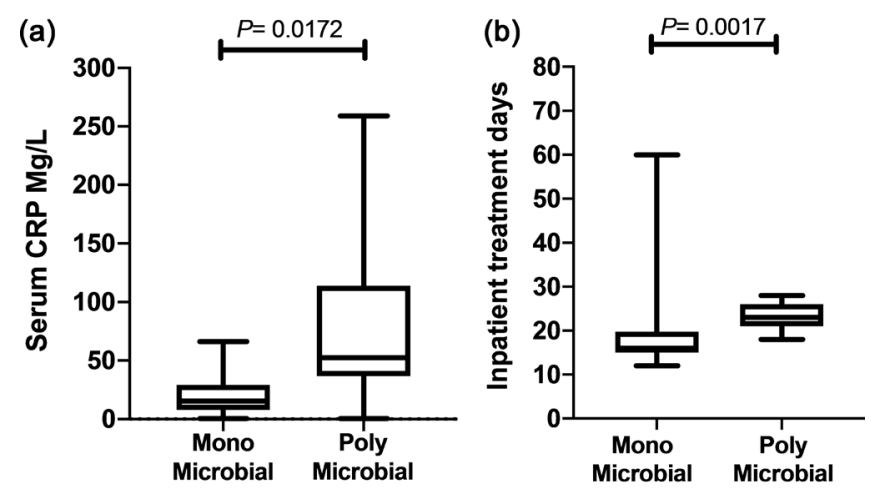

Figure 1. CRP levels and hospitalization days association with single and multiple detected pathogens in sonication culture. (a) CRP serum concentrations of patients with monomicrobial PJI, mean $=20.50, \mathrm{SEM}=3.531$ and patients with polymicrobial PJI, mean $=79.99$, $\mathrm{SEM}=32.47$. (b) Hospitalization treatment days in patients with monomicrobial PJI, mean $=18.71, \mathrm{SEM}=1.907$ and Polymicrobial PJI, mean $=23.39, \mathrm{SEM}=$ 1.267. Monomicrobial $n=24$, Polymicrobial $n=7$. Values shown are median with $\min$ to max values. Mann Whitney test. 
culture $(P=0.0017)$.

\subsection{Highest Congruent Results Found between Intra-Operative and Sonication Culture}

The highest percentage of congruent results were found when comparing intra-operative and sonication cultures, in which 41 patients presented the same findings (concordance of 79\%), Table 8. In 7 cases (13.4\%), the pathogen was detected in sonication only; in 3 patients (5.8\%), the etiological agent was found intraoperatively only and in 7 cases an additional organism was found in sonication. Pre-operative culture congruent results showed a lower concordance percentage when compared with sonication, 31 patients (59.6\%). The pathogen was detected only in sonication in 15 cases (28.8\%); the organism was only identified preoperatively in 5 patients (9.6\%) and an additional pathogen was detected in only 4 cases with the sonication culture. Total pathogen detection congruency between the three groups was seen in 28 patients (54\%).

\subsection{Most of the Patients Presented an Infective Histopathological Classification}

The vast majority of the patients, 36 (69\%) were classified as type II Krenn classification. The second most common classification was type III in 7 cases (13\%), followed by type IV, 5 cases (10\%) and type I in 2 patients (4\%). In two patients the classification was not determined. Since the sonication culture showed the highest pathogen detection percentages, we assessed to compare the microbiological culture findings with the Krenn classification. Type I presented $50 \%$ of positive and negative cultures, type 2 had $69 \%$ of positive results and the rest $31 \%$ of negative cultures findings, type 3 presented a $57 \%$ of positiveness while a $43 \%$ remained negative; type IV showed the lowest positive results with a $20 \%$ and the highest negative microbiological culture findings with a $80 \%$. Table 9 summarizes the histopathological classification and culture findings.

Table 8. Microbiological cultures congruency \%.

\begin{tabular}{cc}
\hline Groups & $\%$ \\
\hline PreOp/Sonication & $31(59.6 \%)$ \\
IntraOp/Sonication & $41(79 \%)$ \\
PreOp/IntraOp/Sonication & $28(54 \%)$ \\
\hline
\end{tabular}

Table 9. Histopathological classification and sonication culture results.

\begin{tabular}{cccc}
\hline Krenn classification & $\mathbf{n}$ & Positive culture & Negative culture \\
\hline I & $4 \%(2)$ & $50 \%(1)$ & $50 \%(1)$ \\
II & $69 \%(36)$ & $69 \%(25)$ & $31 \%(11)$ \\
III & $13 \%(7)$ & $57 \%(4)$ & $43 \%(3)$ \\
IV & $10 \%(5)$ & $20 \%(1)$ & $80 \%(4)$
\end{tabular}




\subsection{Fixation Type Influenced on Microbiological Culture Results}

Cemented fixation was the most commonly used in the patients with a $62 \%$ of the cases, 32 patients, while cement free fixation was found in $37 \%, 19$ cases. As shown in Table 10 microbiological culture results had a trend of presenting higher positive findings in those patients with a cementless fixation in all 3 studied groups when compared to the cemented cases.

\subsection{Total Hip Replacement Arthroplasty Presented the Highest Bacteria Detection by Sonication}

We assessed to compare which of the main affected joints and prostheses type had a higher bacteria detection through sonication culture, Figure 2. Total hip

Table 10. Fixation type and sonication culture results.

\begin{tabular}{cccccc}
\hline \multicolumn{3}{c}{ Cementless 37\% (19) } & & \multicolumn{3}{c}{ Cemented 63\% (32) } \\
\hline \multirow{2}{*}{ Pre-OP } & Positive culture & $53 \%(10)$ & $34 \%(11)$ & Positive culture & Pre-OP \\
& Negative culture & $47 \%(9)$ & $66 \%(21)$ & Negative culture & \\
Intra-OP & Positive culture & $63 \%(12)$ & $44 \%(14)$ & Positive culture & Intra-OP \\
& Negative culture & $37 \%(7)$ & $56 \%(18)$ & Negative culture & \\
Sonication & Positive culture & $74 \%(14)$ & $50 \%(16)$ & Positive culture & Sonication \\
& Negative culture & $26 \%(5)$ & $50 \%(16)$ & Negative culture & \\
\hline
\end{tabular}

(a)
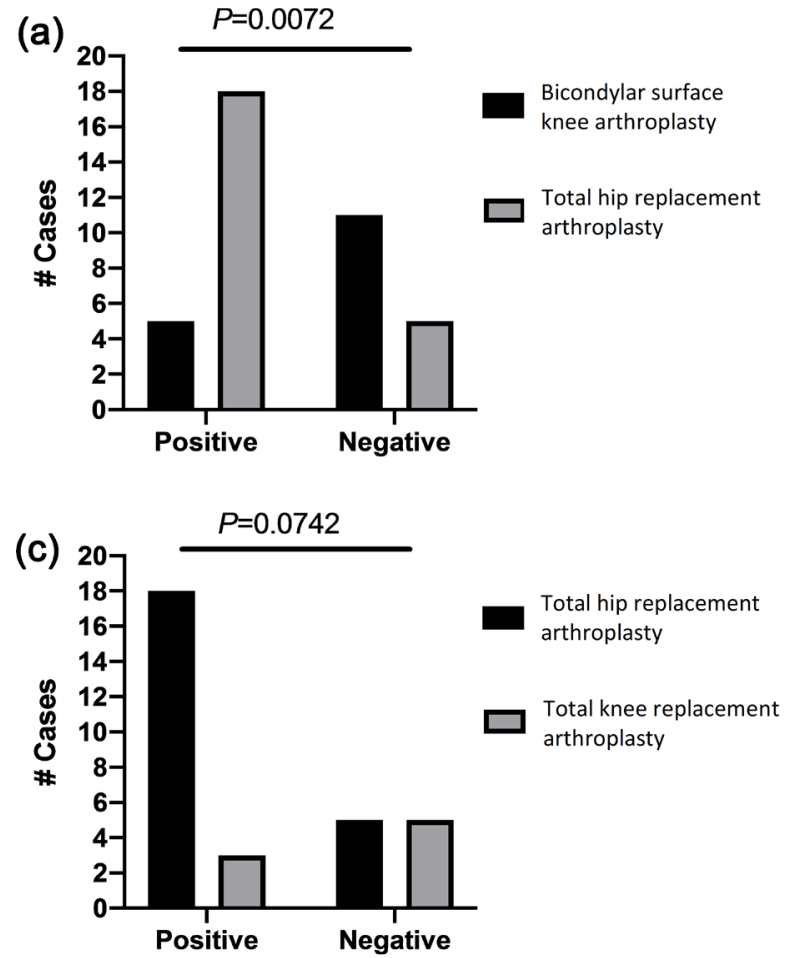

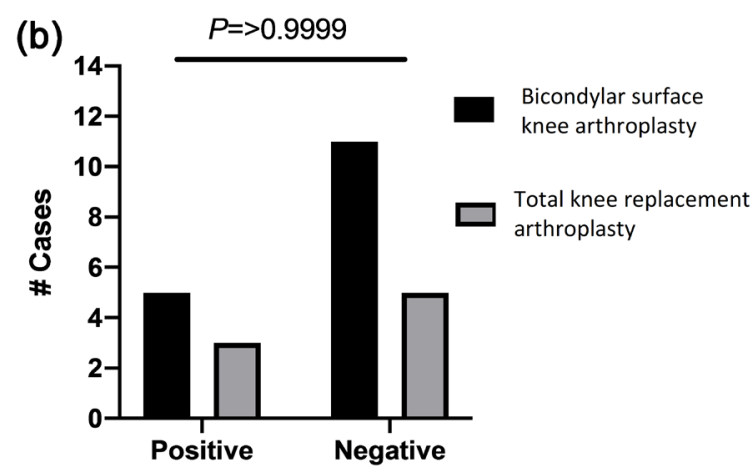

Figure 2. Sonication pathogen detection by joint affection and prostheses type. (a) Bicondylar surface knee arthroplasty $n=16$ and total hip replacement arthroplasty $n=23$. (b) Bicondylar surface knee arthroplasty $n=16$ and total knee replacement arthroplasty $n=8$. (c) Total hip replacement arthroplasty $n=23$ and total knee replacement arthroplasty $n=8$. Fisher's exact test. 
replacement arthroplasty showed the highest pathogen identification percentage, $78.26 \%$, which was statistically significant higher when compared to bicondylar surface knee replacement, $31.25 \%(P=0.0072)$. Besides the fact that there was not a statistically significant difference when compared to total knee replacement $37.50 \%(P=0.0742)$, a trend of a much higher positive bacteria culture result is evident. Bicondylar surface knee arthroplasty and total knee replacement arthroplasty comparison didn't have any difference on the culture results $(P>$ 0.9999). Negative culture results of total hip replacement arthroplasties were of $21.74 \%$, total knee replacement arthroplasties of $62.50 \%$ and bicondylar surface knee replacement arthroplasties of $68.75 \%$.

\section{Discussion}

Low grade PJI is defined as the condition in which there is a lack of acute local inflammatory signs and it is commonly associated with an "unexplained" pain, swelling and reduced functional capacity of the joint which can show up even years after surgery [13]. As seen in our results this infection is usually more common in female population, this in concordance with other studies [15] [16] [17]. However, it has been also reviewd that in some cases male population is considered as a non-modifiable risk factor for developing hip or knee PJI [5] [18]. This gender differences are still uncertain and might be caused due to other factors such as smoking, body mass index and the presence of comorbidities.

It is well known that there is a steady increase in the number of joint replacement operations, in which hip and knee joints are the most common replaced and are expected to rise even more during the next years [19] [20]. As our results shows, these two joints are the most common affected in our studied population, both of them with almost $45 \%$ of the cases. However, something to be considered is the joint affection discrepancy between male and female population. Hip joint was more commonly affected in males while knee joint in females, both of them on more than half of the cases. Despite the fact that this affection behavior has no scientific clarification yet, a possible explanation might be due to anatomically differences between genders which has been reported to make males more susceptible to hip injuries [21] and females to knee injuries due to smaller cartilage volumes [22].

We assessed to compare the performance of sonication culture among standard culture methods of synovial fluid obtained by puncture before operation (Pre-Op) and samples obtained at the moment of operation (Intra-Op). Consistent with other studies [12] [23], sonication culture method appeared to improve PJI microbiological diagnosis in our population, obtaining the highest positive culture percentage (60\%) when compared to the intra-Op (52\%) and pre-Op $(40 \%)$. It has been shown that this method could be particularly useful in those patients in whom surgeons consider 2 stage surgery procedures, in which patients with painful implants, will undergo to implant removal with sonication step followed by a delayed arthroplasty procedure [24]. However, the impor- 
tance of standard culture methods have also been demonstrated and one must take into consideration that those culture methods should probably not be substituted by sonication but combined for obtaining a greater benefit [15] [25]. It has been even described that microbiological diagnosis of PJI improves significantly by combining sonicated fluid in blood culture bottles when compared to just standard tissue cultures, synovial fluid culture, and even synovial fluid PCR for pathogen detection [26].

Around $40 \%$ of PJI infections are due to coagulase negative staphylococci [27] and that overall the most common of them is Staphylococcus epidermidis [28]. This, accordingly to our results in were this etiological agent was the most common isolated pathogen in the 3 groups of cultures. Staphylococcus epidermidis biofilm formation in implanted material has been reviewed and demonstrated in other studies [29] [30] in which the sonication importance is demonstrated by the fact that $99 \%$ of the bacteria resides and remains in the implanted device, cement or any other joint component as bone, tissue or synovial fluid [27], in which a conventional culture alone will not be able to detect the pathogen.

Different from what has been reported, were Staphylococcus aureus is usually the second the most common isolated pathogen in Europe after coagulase negative staphylococci [31], our population showed that it was the third most common etiological agent followed by Propionibacterium acnes. It should be noted that differences between study groups might cause these variations in the culture results, as shown also in USA, in which profile, contrary than Europe, Staphylococcus aureus is the most common isolated pathogen followed by coagulase negative staphylococci [31]. Something to be considered is that Propionibacterium acnes is usually found in patients with persistent chronic pain and loosening of implants and rarely as an acute postoperative infection, this due to the fact that it is a low virulent organism [32] capable of forming biofilms in which sonication culture might be a useful tool for the PJI diagnosis. These were shown in our results in which this pathogen was mainly detected through sonication, and this method presented the highest pathogen detection percentage when all other cultures were negative for a microbiological result.

Approximately $85 \%$ of PJI are caused by a single pathogen, while the rest by polymicrobial infections [33] [34], this accordingly to our results. However, it has been reported that multiple pathogen infections during PJI can be as high as almost 40\% [35]. Our results showed that the highest polymicrobial detection percentage was found in the sonication culture with $14 \%$ of the cases. Among these, no co-pathogens were found more frequently than others, however, the most common isolated pathogen through sonication was Staphylococcus aureus which was found in 3 of the 7 cases. Interestingly, this culture method was not able to detect this pathogen as a single bacterium and was exclusively detected as a co-infective agent. Although little evidence is known about polymicrobial PJI infections, several risk factors have been proposed such as the presence of drainage, soft tissue defect or wound dehiscence, patients over 65 years old [34], presence of 
underlaying fractures and a closer time to the first implant surgery [33].

Since our results showed that sonication culture was best able to detect polymicrobial PJI, we assessed to determine the existence of a clinical relevance of these findings. We found almost 4 times higher serum CRP levels in patients with polymicrobial infections. To our knowledge there is only one other study which found this association [36], however no possible implications where mentioned. CRP also known as C-reactive protein has been used as a nonspecific infection/inflammation marker. But it relevance goes even beyond due to the fact that it plays an important role in activating the complement pathway, apoptosis, phagocytosis, nitric oxide release and the production of important pro-inflammatory cytokines such as IL- 6 and TNF- $\alpha$ [37]. Theoretically, the higher the threat the higher CRP levels, which, will help fight the infection through the inflammatory process. However, these serum concentrations might be also playing a role in a continuous inflammatory state which might keep the patients in a longer hospital stay as shown in our results in which polymicrobial PJI patients had a longer hospitalization period of time. As far as we know this has not been yet associated with PJI inpatient stay, being this the first report, however, it has been previously associated in cardiac operations [38]. Further research is still needed for understanding the clinical impact of these findings.

Based on our results, sonication culture is best able to detect pathogens during low grade PJI. Our data also showed that the closest conventional culture method for getting similar results is through intra-operative samples of synovial fluid or tissue, which had $79 \%$ of concordance with the sonication culture results. Sonication has been proposed to be used specially in patients with delayed implant failure or late infections, in which it presented a higher diagnostic value [39] probably due to the biofilm formation time window. However, it also has been recommended as a routine, cheap and useful test with high sensibility for microbiological diagnosis of PJI in all scenarios [40]. In cases in which hospitals or clinics lack its availability, periprosthetic tissue culture, even when it showed less sensibility than sonication, should be used. On other hand, our pre-operative microbiological results showed the lowest concordance percentage when compared to the sonication culture. These suggests of surgical and antimicrobial treatment of low grade PJI should not be based only in the pre-operative sample cultures.

It should be considered that sonication results might have the risk of contamination at any time while processing the samples. So, we decided to collect also the histological analysis of the periprosthetic membrane of the patients. As showed in our results, most of the patients were classified into a type II or infective category, in which the $69 \%$ were positive for a microorganism, confirming the diagnosis. However, a diagnostic dilemma remained in the rest 39\%, which were classified into this category but had a negative culture finding. Multiple factors might be causing a negative culture with a leukocyte infiltrate. One possible explanation could be that maybe the pathogen was already eliminated by host's immune response at the moment of sampling, were toxins and antigens 
still drives a local inflammatory response [41] [42], or inadequate culture conditions for uncommon pathogens. Our data supports what other study found [43], in which results recommends that when available microbiological and histological findings must be interpreted in combination with the clinical presentation of the patient for a more accurate diagnosis.

The use of antimicrobials in cement for prosthesis is still in discussion. Cement fixation was the most common used in our studied population and our results showed a trend for less positive microbiologic culture results in all preoperative, intraoperative and sonication culture samples when compared to cementless type. Besides there is evidence which states that there is no difference between the use of cement or cementless fixations for developing PJI [44] it has been reviewed that almost $90 \%$ of all cement fixations are made with an antibiotic loaded polymethylmethacrylate [5], being aminoglycosides and glycopeptides the most common ones [45]. This gives a theoretical prevention of PJI despite the lack of high-level evidence which supports this practice.

We assessed to determine which type of protheses had higher bacteria detection through sonication culture. Our results showed that total hip replacement arthroplasty had the highest pathogen detection percentage among total knee replacement arthroplasty and bicondylar surface knee arthroplasty. This is something to be consider for identifying optimal infection preventive measures depending on the patients characteristics, type of surgery and its complexity which have been associated with an increased risk of infection [46] [47].

As with most of research studies, our retrospective study design is subject to certain limitations. One possible limitation was the relatively small number of patients included in the research, as well as that the study was mainly focused on hip and knee arthroplasties. No molecular diagnosis of low grade PJI was made which would have been interesting to include for confirming a bacteria presence.

\section{Conclusion}

In summary, our study showed that sonication culture could help in the low grade PJI microbiological diagnosis presenting a higher pathogen detection percentage for monomicrobial and polymicrobial infections. With this method, we were able to detect that polymicrobial infections may impact on patient's hospitalization days and CRP levels. The prosthesis design and cemented fixation techniques seem to have an influence on the sonication result. There is a high degree of agreement between the histopathological detection of infection in the classification according to Krenn and the detection of bacteria in sonication. Therefore, in addition to the microbiological parameters, histological and clinical parameters must always be considered in order to achieve the most accurate diagnosis of PJI.

\section{Acknowledgements}

JAGC would like to thank CONACYT (Consejo Nacional de Ciencia y Tecnolo- 
gia) for the given scholarship 708282 .

\section{Conflicts of Interest}

The authors declare no conflicts of interest regarding the publication of this paper.

\section{References}

[1] Parvizi, J., Gehrke, T. and Chen, A.F. (2013) Proceedings of the International Consensus on Periprosthetic Joint Infection. The Bone \& Joint Journal, 95-B, 1450-1452. https://doi.org/10.1302/0301-620X.95B11.33135

[2] Parvizi, J., Zmistowski, B., Berbari, E.F., Bauer, T.W., Springer, B.D., Della Valle, C.J., et al. (2011) New Definition for Periprosthetic Joint Infection: From the Workgroup of the Musculoskeletal Infection Society. Clinical Orthopaedics and Related Research, 469, 2992-2994. https://doi.org/10.1007/s11999-011-2102-9

[3] Li, C., Renz, N. and Trampuz, A. (2018) Management of Periprosthetic Joint Infection. Hip Pelvis, 30, 138-146. https://doi.org/10.5371/hp.2018.30.3.138

[4] Romano, C.L., Romano, D., Morelli, I. and Drago, L. (2017) The Concept of Biofilm-Related Implant Malfunction and "Low-Grade Infection". Advances in Experimental Medicine and Biology, 971, 1-13. https://doi.org/10.1007/5584 2016158

[5] Tande, A.J. and Patel, R. (2014) Prosthetic Joint Infection. Clinical Microbiology Reviews, 27, 302-345. https://doi.org/10.1128/CMR.00111-13

[6] Donlan, R.M. (2002) Biofilms: Microbial Life on Surfaces. Emerging Infectious Diseases, 8, 881-890. https://doi.org/10.3201/eid0809.020063

[7] Khatoon, Z., McTiernan, C.D., Suuronen, E.J., Mah, T.F. and Alarcon, E.I. (2018) Bacterial Biofilm Formation on Implantable Devices and Approaches to Its Treatment and Prevention. Heliyon, 4, e01067. https://doi.org/10.1016/j.heliyon.2018.e01067

[8] Flemming, H.C., Wingender, J., Szewzyk, U., Steinberg, P., Rice, S.A. and Kjelleberg, S. (2016) Biofilms: An Emergent Form of Bacterial Life. Nature Reviews Microbiology, 14, 563-575. https://doi.org/10.1038/nrmicro.2016.94

[9] Palan, J., Nolan, C., Sarantos, K., Westerman, R., King, R. and Foguet, P. (2019) Culture-Negative Periprosthetic Joint Infections. EFORT Open Reviews, 4, 585-594. https://doi.org/10.1302/2058-5241.4.180067

[10] Ehrlich, G.D., et al. (2012) Culture-Negative Infections in Orthopedic Surgery. In: Ehrlich, G.D., Costerton, J.W. and Winkler, H., Eds., Culture Negative Orthopedic Biofilm Infections, Springer, Berlin, Heidelberg, 17-27.

https://doi.org/10.1007/978-3-642-29554-6 2

[11] Erivan, R., Villatte, G., Eymond, G., Mulliez, A., Descamps, S. and Boisgard, S. (2018) Usefulness of Sonication for Diagnosing Infection in Explanted Orthopaedic Implants. Orthopaedics \& Traumatology, Surgery \& Research: OTSR, 104, 433-438. https://doi.org/10.1016/j.otsr.2017.11.021

[12] Abou El-Khier, N.T., El-Kazzaz, S.S., Elgeidi, A. and Elganainy, A.E.R. (2019) Sonication to Improve the Yield in Culture-Negative Peri-Prosthetic Joint Infection. Egyptian Journal of Basic and Applied Sciences, 6, 46-53. https://doi.org/10.1080/2314808X.2019.1651552

[13] Romano, C.L., Khawashki, H.A., Benzakour, T., Bozhkova, S., Del Sel, H., Hafez, M., et al. (2019) The W.A.I.O.T. Definition of High-Grade and Low-Grade Peri-Prosthetic Joint Infection. Journal of Clinical Medicine, 8, 650. 
https://doi.org/10.3390/jcm8050650

[14] Morawietz, L., Classen, R.A., Schroder, J.H., Dynybil, C., Perka, C., Skwara, A., et al. (2006) Proposal for a Histopathological Consensus Classification of the Periprosthetic Interface Membrane. Journal of Clinical Pathology, 59, 591-597. https://doi.org/10.1136/jcp.2005.027458

[15] Shen, H., Tang, J., Wang, Q., Jiang, Y. and Zhang, X. (2015) Sonication of Explanted Prosthesis Combined with Incubation in BD Bactec Bottles for Pathogen-Based Diagnosis of Prosthetic Joint Infection. Journal of Clinical Microbiology, 53, 777-781. https://doi.org/10.1128/JCM.02863-14

[16] Pulido, L., Ghanem, E., Joshi, A., Purtill, J.J. and Parvizi, J. (2008) Periprosthetic Joint Infection: The Incidence, Timing, and Predisposing Factors. Clinical Orthopaedics and Related Research, 466, 1710-1715. https://doi.org/10.1007/s11999-008-0209-4

[17] Li, Z.L., Hou, Y.F., Zhang, B.Q., Chen, Y.F., Wang, Q., Wang, K., et al. (2018) Identifying Common Pathogens in Periprosthetic Joint Infection and Testing Drug-Resistance Rate for Different Antibiotics: A Prospective, Single Center Study in Beijing. Orthopaedic Surgery, 10, 235-240. https://doi.org/10.1111/os.12394

[18] Lenguerrand, E., Whitehouse, M.R., Beswick, A.D., Kunutsor, S.K., Burston, B., Porter, M., et al. (2018) Risk Factors Associated with Revision for Prosthetic Joint Infection after Hip Replacement: A Prospective Observational Cohort Study. The Lancet Infectious Diseases, 18, 1004-1014. https://doi.org/10.1016/S1473-3099(18)30345-1

[19] Kurtz, S., Ong, K., Lau, E., Mowat, F. and Halpern, M. (2007) Projections of Primary and Revision Hip and Knee Arthroplasty in the United States from 2005 to 2030. The Journal of Bone and Joint Surgery American Volume, 89, 780-785. https://doi.org/10.2106/00004623-200704000-00012

[20] Kurtz, S.M., Lau, E., Schmier, J., Ong, K.L., Zhao, K. and Parvizi, J. (2008) Infection Burden for Hip and Knee Arthroplasty in the United States. The Journal of Arthroplasty, 23, 984-991. https://doi.org/10.1016/j.arth.2007.10.017

[21] Stewart, C., Wang, C.B., Lange, D., Poster, C.S., Lange, A.W., Kohoyda-Inglis, C., Sochor, M.R., Ipaktchi, K. and Rowe, S.A. (2004) Gender Differences in Hip Anatomy: Possible Implications for Injury Tolerance in Frontal Collisions. Annual Proceedings of the Association for the Advancement of Automotive Medicine, 48, 287-301.

[22] Faber, S.C., et al. (2001) Gender Differences in Knee Joint Cartilage Thickness, Volume and Articular Surface Areas: Assessment with Quantitative Three-Dimensional MR Imaging. Skeletal Radiology, 30, 144-150. https://doi.org/10.1007/s002560000320

[23] Trampuz, A., Piper, K.E., et al. (2007) Sonication of Removed Hip and Knee Prostheses for Diagnosis of Infection. The New England Journal of Medicine, 357, 654-663. https://doi.org/10.1056/NEJMoa061588

[24] Maniar, H.H., Wingert, N., McPhillips, K., Foltzer, M., Graham, J., Bowen, T.R. and Horwitz, D.S. (2016) Role of Sonication for Detection of Infection in Explanted Orthopaedic Trauma Implants. Journal of Orthopaedic Trauma, 30, e175-e180. https://doi.org/10.1097/BOT.0000000000000512

[25] Dudareva, M., Figtree, M., Scarborough, M., Watanabe, M., Newnham, R., Wallis, R., Oakley, S., Kendrick, B., Stubbs, D., McNally, M.A., Bejon, P., Atkins, B.A., Taylor, A. and Brent, A.J. (2018) Sonication versus Tissue Sampling for Diagnosis of Prosthetic Joint and Other Orthopedic Device-Related Infections. Journal of Clinical Microbiology, 56, e00688-18. https://doi.org/10.1128/JCM.00688-18 
[26] Li, C., Renz, N., Thies, C.O. and Trampuz, A. (2018) Meta-Analysis of Sonicate Fluid in Blood Culture Bottles for Diagnosing Periprosthetic Joint Infection. Journal of Bone and Joint Infection, 3, 273-279. https://doi.org/10.7150/jbji.29731

[27] McConoughey, S.J., Howlin, R., Granger, J.F., Manring, M.M., Calhoun, J.H., Shirtliff, M., et al. (2014) Biofilms in Periprosthetic Orthopedic Infections. Future Microbiology, 9, 987-1007. https://doi.org/10.2217/fmb.14.64

[28] Becker, K., Heilmann, C. and Peters, G. (2014) Coagulase-Negative Staphylococci. Clinical Microbiology Reviews, 27, 870-926. https://doi.org/10.1128/CMR.00109-13

[29] Hellmark, B., Soderquist, B., Unemo, M. and Nilsdotter-Augustinsson, A. (2013) Comparison of Staphylococcus epidermidis Isolated from Prosthetic Joint Infections and Commensal Isolates in Regard to Antibiotic Susceptibility, Agr Type, Biofilm Production, and Epidemiology. International Journal of Medical Microbiology: IJMM, 303, 32-39. https://doi.org/10.1016/j.ijmm.2012.11.001

[30] Fey, P.D. and Olson, M.E. (2010) Current Concepts in Biofilm Formation of Staphylococcus epidermidis. Future Microbiology, 5, 917-933. https://doi.org/10.2217/fmb.10.56

[31] Aggarwal, V.K., Bakhshi, H., Ecker, N.U., Parvizi, J., Gehrke, T. and Kendoff, D. (2014) Organism Profile in Periprosthetic Joint Infection: Pathogens Differ at Two Arthroplasty Infection Referral Centers in Europe and in the United States. Journal of Knee Surgery, 27, 399-406. https://doi.org/10.1055/s-0033-1364102

[32] Rienmuller, A. and Borens, O. (2016) Propionibacterium Prosthetic Joint Infection: Experience from a Retrospective Database Analysis. European Journal of Orthopaedic Surgery \& Traumatology: Orthopedie Traumatologie, 26, 429-434. https://doi.org/10.1007/s00590-016-1766-y

[33] Flurin, L., Greenwood-Quaintance, K.E. and Patel, R. (2019) Microbiology of Polymicrobial Prosthetic Joint Infection. Diagnostic Microbiology and Infectious Disease, 94, 255-259. https://doi.org/10.1016/j.diagmicrobio.2019.01.006

[34] Marculescu, C.E. and Cantey, J.R. (2008) Polymicrobial Prosthetic Joint Infections: Risk Factors and Outcome. Clinical Orthopaedics and Related Research, 466, 1397-1404. https://doi.org/10.1007/s11999-008-0230-7

[35] Moran, E., Masters, S., Berendt, A.R., McLardy-Smith, P., Byren, I. and Atkins, B.L. (2007) Guiding Empirical Antibiotic Therapy in Orthopaedics: The Microbiology of Prosthetic Joint Infection Managed by Debridement, Irrigation and Prosthesis Retention. The Journal of Infection, 55, 1-7. https://doi.org/10.1016/j.jinf.2007.01.007

[36] Bozhkova, S., Tikhilov, R., Labutin, D., Denisov, A., Shubnyakov, I., Razorenov, V., et al. (2016) Failure of the First Step of Two-Stage Revision Due to Polymicrobial Prosthetic Joint Infection of the Hip. Journal of Orthopaedics and Traumatology, 17, 369-376. https://doi.org/10.1007/s10195-016-0417-8

[37] Sproston, N.R. and Ashworth, J.J. (2018) Role of C-Reactive Protein at Sites of Inflammation and Infection. Frontiers in Immunology, 9, 754.

https://doi.org/10.3389/fimmu.2018.00754

[38] Fransen, M.E.J., Maessen, J.G., Elenbaas, T.W.O., van Aarnhem, E.E.H.L. and van Dieijen-Visser, M.P. (1999) Increased Preoperative C-Reactive Protein Plasma Levels as a Risk Factor for Postoperative Infections. The Annals of Thoracic Surgery, 67, 134-138. https://doi.org/10.1016/S0003-4975(98)00973-4

[39] Puig-Verdié, L., González-Cuevas, A., Sorlí, L., Salvadó, M., Alier, A., Pelfort, X., Portillo, M.E. and Horcajada, J.P. (2013) Implant Sonication Increases the Diagnostic Accuracy of Infection in Patients with Delayed, But Not Early, Orthopaedic Implant Failure. The Bone \& Joint Journal, 95-B, 244-249. 
https://doi.org/10.1302/0301-620X.95B2.30486

[40] Tani, S., Lepetsos, P., Stylianakis, A., Vlamis, J., Birbas, K. and Kaklamanos, I. (2018) Superiority of the Sonication Method against Conventional Periprosthetic Tissue Cultures for Diagnosis of Prosthetic Joint Infections. European Journal of Orthopaedic Surgery \& Traumatology: Orthopedie Traumatologie, 28, 51-57. https://doi.org/10.1007/s00590-017-2012-y

[41] Ali, A., Zhu, X., Kwiecinski, J., Gjertsson, I., Lindholm, C., Iwakura, Y., et al. (2015) Antibiotic-Killed Staphylococcus aureus Induces Destructive Arthritis in Mice. Arthritis \& Rheumatology, 67, 107-116. https://doi.org/10.1002/art.38902

[42] Noorbakhsh, S. and Tabatabaei, A. (2013) Identification of Bacterial Antigens and Super Antigens in Synovial Fluid of Patients with Arthritis: A Cross Sectional Study. Medical Journal of Islamic Republic of Iran, 27, 12-16.

[43] Janz, V., Wassilew, G.I., Hasart, O., Matziolis, G., Tohtz, S. and Perka, C. (2013) Evaluation of Sonicate Fluid Cultures in Comparison to Histological Analysis of the Periprosthetic Membrane for the Detection of Periprosthetic Joint Infection. International Orthopaedics, 37, 931-936. https://doi.org/10.1007/s00264-013-1853-1

[44] Jamsen, E., Huhtala, H., Puolakka, T. and Moilanen, T. (2009) Risk Factors for Infection after Knee Arthroplasty. A Register-Based Analysis of 43,149 Cases. The Journal of Bone and Joint Surgery American Volume, 91, 38-47. https://doi.org/10.2106/JBJS.G.01686

[45] Anagnostakos, K. (2017) Therapeutic Use of Antibiotic-Loaded Bone Cement in the Treatment of Hip and Knee Joint Infections. Journal of Bone and Joint Infection, 2, 29-37. https://doi.org/10.7150/jbji.16067

[46] Korol, E., Johnston, K., Waser, N., Sifakis, F., Jafri, H.S., Lo, M., et al. (2013) A Systematic Review of Risk Factors Associated with Surgical Site Infections among Surgical Patients. PLoS ONE, 8, e83743. https://doi.org/10.1371/journal.pone.0083743

[47] Cheng, H., Clymer, J.W., Po-Han Chen, B., Sadeghirad, B., Ferko, N.C., Cameron, C.G., et al. (2018) Prolonged Operative Duration Is Associated with Complications: A Systematic Review and Meta-Analysis. Journal of Surgical Research, 229, 134-144. https://doi.org/10.1016/j.jss.2018.03.022 


\section{Supplementary}

Table S1. Microbiological findings in all patients.

\begin{tabular}{|c|c|c|c|c|c|}
\hline Pre-OP & Sensible & Intra-OP & Sensible & Sonication & Sensible \\
\hline Propionibacterium acnes & MS & - & - & Propionibacterium acnes & MS \\
\hline- & - & - & - & - & - \\
\hline- & - & - & - & Propionibacterium acnes & - \\
\hline- & - & - & - & - & - \\
\hline Enterococcus faecalis & MS & $\begin{array}{l}\text { Enterococcus faecalis Staphylococcus } \\
\text { capitis Staphylococcuswarneri }\end{array}$ & MS & Enterococcus faecalis & - \\
\hline \multirow[t]{2}{*}{-} & - & $\begin{array}{l}\text { Staphylococcus epidermidis } \\
\text { Streptococcus sanguinis }\end{array}$ & MS & Streptococcus sanguinis & MS \\
\hline & - & - & - & - & - \\
\hline Staphylococcus aureus & MS & Staphylococcus aureus & MS & $\begin{array}{l}\text { Staphylococcus aureus } \\
\text { Staphylococcus caprae }\end{array}$ & MS \\
\hline Staphylococcus epidermidis & MR & - & - & Staphylococcus epidermidis & MR \\
\hline Propionibacterium acnes & MS & - & - & - & - \\
\hline- & - & - & - & - & - \\
\hline - & - & Staphylococcus epidermidis & MS & - & - \\
\hline - & - & - & - & - & - \\
\hline- & - & - & - & Staphylococcus haemolyticus & MS \\
\hline Staphylococcus epidermidis & MS & Staphylococcus epidermidis & MR & Staphylococcus epidermidis & MR \\
\hline Propionibacterium acnes & MS & Propionibacterium acnes & MS & Propionibacterium acnes & MS \\
\hline- & - & - & - & - & - \\
\hline- & - & - & - & - & - \\
\hline Staphylococcus epidermidis & MR & - & - & - & - \\
\hline- & - & - & - & - & - \\
\hline- & - & Staphylococcus epidermidis & MR & $\begin{array}{l}\text { Staphylococcus epidermidis } \\
\text { Staphylococcus haemolyticus }\end{array}$ & MR \\
\hline $\begin{array}{l}\text { Staphylococcus epidermidis } \\
\text { Staphyloccucus caprae }\end{array}$ & MR & Staphylococcus epidermidis & MR & Staphylococcus epidermidis & MR \\
\hline Staphylococcus epidermidis & MR & Staphylococcus epidermidis & MR & Staphylococcus epidermidis & MR \\
\hline $\begin{array}{l}\text { Pseudomonas aeruginosa, } \\
\text { Klebsiella aerogenes }\end{array}$ & MS & Pseudomonas aeruginosa & - & - & - \\
\hline- & - & - & - & - & - \\
\hline Staphylococcus epidermidis & MR & Staphylococcus epidermidis & MR & $\begin{array}{l}\text { Staphylococcus epidermidis } \\
\text { Propionibacterium avidum }\end{array}$ & - \\
\hline - & - & - & - & Propionibacterium acnes & MS \\
\hline Staphylococcus epidermidis & MS & Staphylococcus epidermidis & MS & Staphylococcus epidermidis & MS \\
\hline Staphylococcus capitis & MR & $\begin{array}{l}\text { Staphylococcus capitis } \\
\text { Propionibacterium acnes }\end{array}$ & MR & Staphylococcus capitis & MR \\
\hline Staphylococcus aureus & MS & Staphylococcus aureus & MS & Staphylococcus aureus Bacillus cereus & MS \\
\hline
\end{tabular}


N. Haustedt et al.

\section{Continued}

\begin{tabular}{|c|c|c|c|c|c|}
\hline Enterococcus faecalis & MS & - & - & - & - \\
\hline Streptococcus mitis & MS & Streptococcus mitis & MS & - & - \\
\hline- & - & Staphylococcus epidermidis & MR & Staphylococcus epidermidis & MR \\
\hline Pseudomonas aeruginosa & TR & Pseudomonas aeruginosa & TR & $\begin{array}{l}\text { Pseudomonas } \\
\text { aeruginosaStaphylococcus } \\
\text { haemolyticus }\end{array}$ & - \\
\hline- & - & - & - & - & - \\
\hline- & - & - & - & - & - \\
\hline- & - & Staphylococcus aureus & MS & $\begin{array}{l}\text { Staphylococcus aureus, Streptococcus } \\
\text { oralis }\end{array}$ & MS \\
\hline Propionibacterium acnes & MS & Propionibacterium acnes & MS & Propionibacterium acnes & MS \\
\hline - & - & - & - & - & - \\
\hline Staphylococcus epidermidis & - & Staphylococcus epidermidis & MS & Staphylococcus epidermidis & MS \\
\hline- & - & - & - & - & - \\
\hline- & - & Staphylococcus epidermidis & MR & Staphylococcus epidermidis & MR \\
\hline Propionibacterium acnes & MS & Actiomyces israelii & MS & $\begin{array}{l}\text { Propionibacterium acnes, } \\
\text { Actinomyces israelii }\end{array}$ & MS \\
\hline- & - & - & - & Propionibacterium acnes & MS \\
\hline- & - & - & - & Propionibacterium acnes & MS \\
\hline- & - & Staphylococcus lugdunensis & MS & Staphylococcus lugdunensis & MS \\
\hline- & - & Staphylococcus epidermidis & MS & Staphylococcus epidermidis & MS \\
\hline- & - & Propionibacterium acnes & MS & Propionibacterium acnes & MS \\
\hline- & - & Propionibacterium acnes & MS & Propionibacterium acnes & MS \\
\hline- & - & - & - & - & - \\
\hline - & - & Staphylococcus epidermidis & MR & Staphylococcus epidermidis & MR \\
\hline
\end{tabular}

